\title{
Catalyst-Free Growth of High-Quality Graphene by High- Temperature Plasma Reaction
}

\author{
Toshiaki Kato*, Masato Morikawa, Hiroo Suzuki, Bin Xu, Rikizo \\ Hatakeyama, and Toshiro Kaneko \\ Department of Electronic Engineering, Tohoku University, Japan
}

Received: November 16, 2013; Accepted: December 19, 2013; Published: December 20, 2013

"Corresponding author: Toshiaki Kato, Department of Electronic Engineering, Tohoku University, Japan, Tel: +81-22-795-7046; Fax: +81-22-2639225; E-mail: kato12@ecei.tohoku.ac.jp

\begin{abstract}
Impurity-free and high-quality monolayered graphene was grown directly on a $\mathrm{SiO}_{2}$ substrate by catalyst-free plasma chemical vapor deposition (CVD). Raman spectroscopy mapping revealed that the D-peak intensity of the synthesized graphene was lower than the detection limit, and that its $2 \mathrm{D} / \mathrm{G}$ intensity ratio was high $(\sim 10)$. Because the D-peak intensity and 2D/G peak ratio of graphene are reflective of its defect density, number of layers, and impurity density, these results indicated that the graphene grown by the investigated method had a monolayered structure with low defect and impurity densities. Systematic investigations also elucidated the optimal conditions for the catalyst-free growth of graphene. It was found that a relatively higher temperature $\left(\sim 975^{\circ} \mathrm{C}\right)$ is necessary to realize the catalyst-free growth of graphene compared with plasma CVD growth using a catalyst $\left(600-900^{\circ} \mathrm{C}\right)$. Low $\mathrm{H}_{2}$ concentration was also found to be critical for growing high-quality graphene. Because the investigated method allowed the growth of high-quality, impurityfree monolayered graphene directly on an insulating substrate without requiring a catalyst, it should contribute to the fabrication of high-performance graphene-based electrical and biomedical devices.
\end{abstract}

Keywords: Graphene; Catalyst free; High quality; Plasma CVD

\section{Introduction}

Graphene is a monolayered carbon sheet in which the carbon atoms are arranged in a hexagonal network. Because graphene exhibits excellent electrical, mechanical, and optical characteristics [1,2], it has come to be regarded as one of the most promising materials for the fabrication of highperformance, flexible electrical devices such as thin-film transistors, transparent conductive films, and various types of sensors [3-5]. In general, graphene-based electrical devices are fabricated by the following processes. First, graphene is grown on the surface of a metallic substrate such as $\mathrm{Ni}$ and $\mathrm{Cu}$ by thermal chemical vapor deposition (CVD) [6]. Then, it is transferred to an insulating substrate by a conventional polymer capping method [4]. This process enables one to prepare meterscale, large graphene sheets on the insulating substrate, which is useful as a starting material for device fabrication. However, this transfer process sometimes induces a number of kinks and introduces various impurities in the synthesized graphene, which significantly deteriorate its mobility and conductivity, resulting in poor device performance. The direct growth of graphene on an insulating substrate is a promising method for overcoming this issue. Recently, we had reported the direct growth of high-quality graphene and graphene nanoribbons on a $\mathrm{SiO}_{2}$ substrate by rapid-heating plasma CVD $[7,8]$. In this method, a catalyst is necessary to realize the nucleation of graphene. However, any remaining catalyst can decrease the stability of graphene-based devices owing to its slow oxidation. Further, in order to be suitable for biomedical applications, graphene must be impurity free because nanoparticles of transition metals are known to be harmful to humans. In spite of the importance of using impurity-free graphene, the catalyst-free growth of highquality monolayered graphene has not yet been realized, with only multilayered nanographene being synthesized successfully without a catalyst [9]. Very recently, relatively novel approach is reported based on $\mathrm{Cu}$ vapor assisted graphene growth [10].

Here, we report the catalyst-free growth of high-quality monolayered graphene on an insulating substrate by high temperature plasma CVD. Raman spectroscopy mapping revealed that the ratio of the $2 \mathrm{D} / \mathrm{G}$ peak intensities of the synthesized graphene was high $(\sim 10)$ while the D-peak intensity was lower than the detection limit. These results indicate that high-quality graphene can be grown directly on an insulating substrate without a catalyst. The optimal conditions for the catalyst-free growth of graphene by plasma CVD were also determined systematically.

\section{Methods}

Graphene was grown using a laboratory-made plasma CVD system $[7,8]$. A mixture of methane and hydrogen gases was used as the carbon source. An inductively coupled plasma was generated by supplying radio frequency $(\mathrm{rf})$ power $\left(\mathrm{P}_{\mathrm{RF}}\right)(0-110$ W) at $13.56 \mathrm{MHz}$ to the coils, which were set outside the quartz tube. The growth temperature $\left(900-1000{ }^{\circ} \mathrm{C}\right)$ was controlled with a conventional electrical furnace. The structure of the synthesized graphene was analyzed using optical microscopy, Raman scattering spectroscopy performed with an $\operatorname{Ar}(488 \mathrm{~nm}$ wavelength) laser (Horiba, HR800), scanning electron microscopy 
(SEM) (Hitachi, SU1510), and atomic force microscopy (AFM) (JEOL, JSPM-5400).

\section{Results and Discussion}

Figure 1 shows the typical SEM (Figure. 1a, b) and AFM (Figure. 1c) images of the synthesized graphene and Raman mapping images showing the intensities of the integrated D-band $\left(\mathrm{I}_{\mathrm{D}}\right.$ ) (Figure. 1d), G-band $\left(\mathrm{I}_{\mathrm{G}}\right.$ ) (Figure. 1e), and 2D-band $\left(\mathrm{I}_{2 \mathrm{D}}\right)$ (Figure. 1f), as well as the $2 \mathrm{D} / \mathrm{G}\left(\mathrm{I}_{2 \mathrm{D}} / \mathrm{I}_{\mathrm{G}}\right)$ intensity ratio (Figure. $1 \mathrm{~g})$. Graphene islands $(\sim 20 \mu \mathrm{m})$ could be directly grown on the surface of $\mathrm{SiO}_{2}$ substrate without using a catalyst. The $\mathrm{I}_{2 \mathrm{D}} /$ $I_{G}$ ratio and the shape of the $2 \mathrm{D}$ spectrum of a graphene sample are known to be indicative of the number of layers of the sample. Monolayered graphene exhibits an $\mathrm{I}_{2 \mathrm{D}} / \mathrm{I}_{\mathrm{G}}$ ratio higher than $\sim 2$ and its 2D peak can be fitted using a single Lorentzian curve [11]. (Figure. 1h) shows the raw Raman scattering spectra of the synthesized graphene; the spectra were obtained from positions $\# 1, \# 2$, and \#3 in (Figure. 1g). The layer number of graphene can be judged from the $\mathrm{I}_{2 \mathrm{D}} / \mathrm{I}_{\mathrm{G}}$ ratio values and the shape of the 2D raw spectra, which reveal that the positions \#1, \#2, and \#3 in (Figure. 1g) corresponded to few-layered, double-layered, and monolayered graphene, respectively. Interestingly, the $\mathrm{I}_{2 \mathrm{D}} /$ $\mathrm{I}_{\mathrm{G}}$ ratio at \#3 was significantly high $(\sim 10)$ and much higher than that usually noticed for monolayered graphene $(\sim 2)$ (Figure. 1i). Because the value of $I_{2 D}$ is reflective of the doping density of graphene [11], higher 2D peak intensity would indicate a lower doping density that is a lower impurity density. Furthermore, the values of $I_{D}$ for the peaks attributable to the defects in the graphene sample were below the detection limit for almost every position in (Figure. 1d). This indicates that the graphene sample grown directly on an insulating substrate without a catalyst by high-temperature-plasma CVD was impurity free and of very high quality.

Systematic investigations are also carried out to identify the optimal conditions for growing graphene using hightemperature-plasma CVD. (Figure. 2) shows the 2D/G intensity as the functions of the growth temperature (Figure. 2a), growth time (Figure. $2 \mathrm{~b}$ ), $\mathrm{H}_{2}$ concentration (Figure. $2 \mathrm{c}$ ), and $\mathrm{P}_{\mathrm{RF}}$ for plasma generation (Figure. 2d). The curves indicate that a relatively high temperature and low $\mathrm{H}_{2}$ concentration are optimal for the catalyst-free growth of graphene. The growth-time dependence curve, shown in (Figure. 2b) also yielded useful information
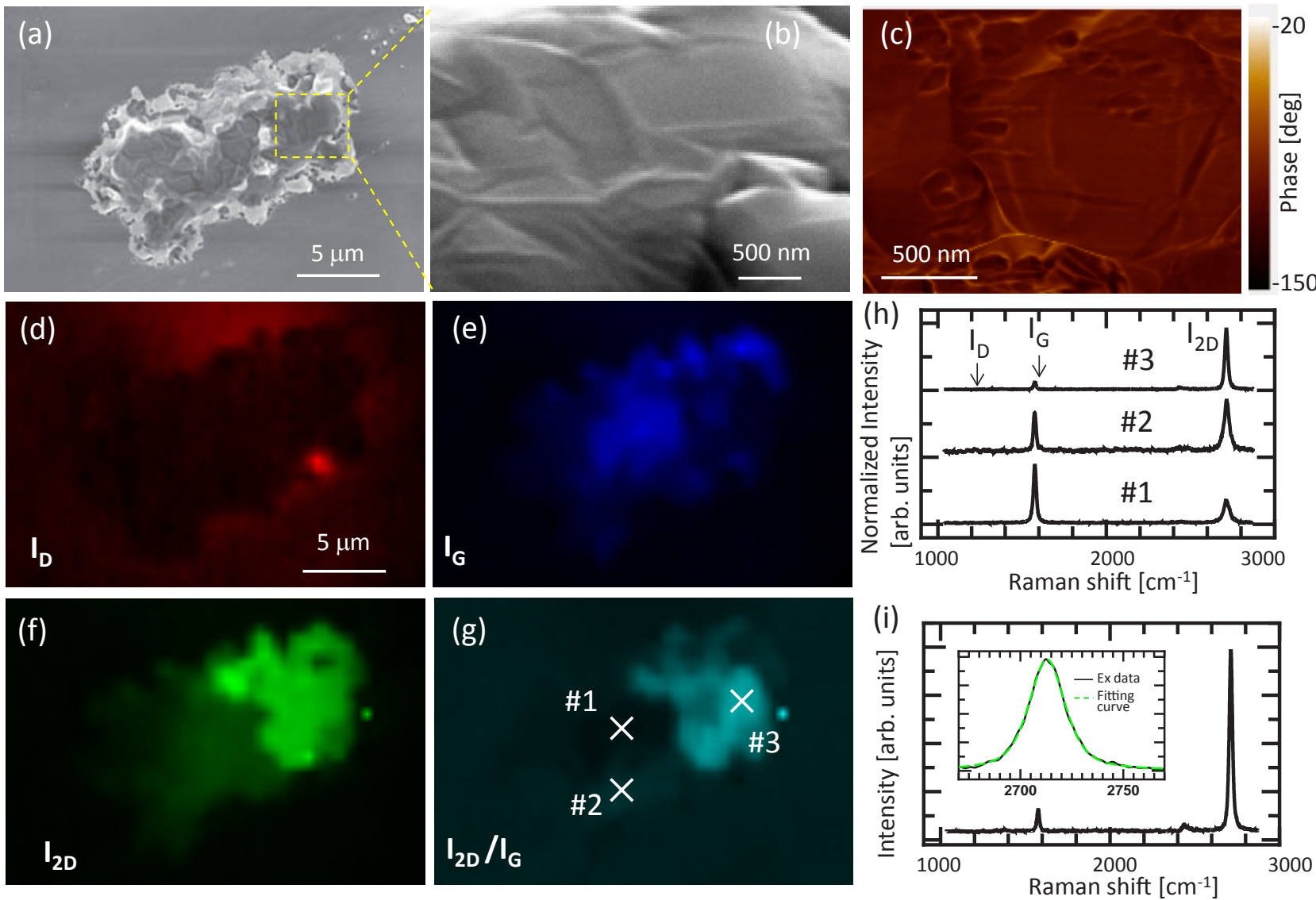

Figure 1: (a) Low and (b) high-magnification SEM images and (c) AFM phase image of graphene grown directly on a $\mathrm{SiO}_{2}$ substrate without a catalyst. (d-g) Raman mapping images of the (d) D-peak, (e) G-peak, and (f) 2D-peak, as well as the (g) 2D/G intensity ratio. The same graphene sample was used for (a) SEM imaging and (d-g) Raman mapping. (h) Typical raw Raman scattering spectra of graphene obtained at positions \#1, \#2, and \#3 in (g). Each peak was normalized by the maximum intensity. (i) Raw Raman scattering spectra of monolayered graphene obtained at position \#3. Inset shows the magnified 2D spectrum (black) and curve (green) fitted with a single Lorentzian function. 

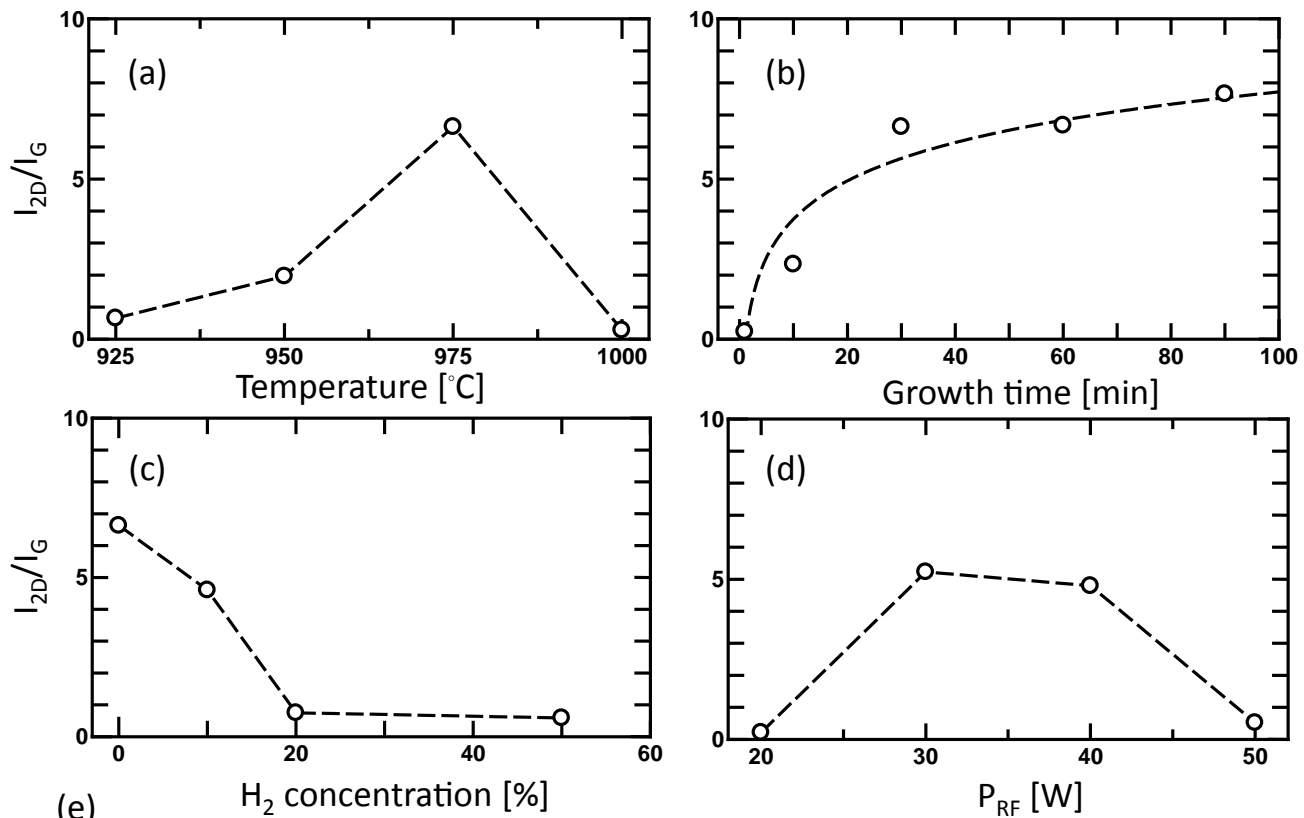

(e)

$\mathrm{H}_{2}$ concentration [\%]

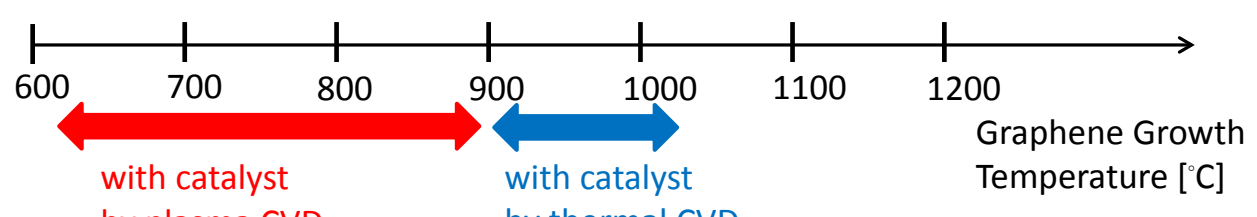

by plasma CVD

by thermal CVD

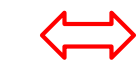

without catalyst

by plasma CVD

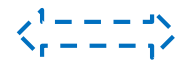

without catalyst

by thermal CVD?

Figure 2: Plots of $\mathrm{I}_{2 \mathrm{D}} / \mathrm{I}_{\mathrm{G}}$ as functions of the (a) growth temperature, (b) growth time, (c) $\mathrm{H}_{2}$ concentration, and (d) $\mathrm{P}_{\mathrm{RF}}$ ( (e) Comparison of the optimal temperatures and methods for graphene growth.

regarding the carbon diffusion kinetics [12]. In the case of a diffusion reaction on the surface of a material such as $\mathrm{Cu}$, the number of synthesized graphene layers does not increase with an increase in the growth time; this is because the diffusion reaction is self-limiting [13]. On the other hand, thick multilayered graphene is grown for long growth times in the case of substrates such as $\mathrm{Ni}$, as in such cases, the diffusion reaction is a bulk one [4]. As shown in (Figure. 2b), the value of $\mathrm{I}_{2 \mathrm{D}} / \mathrm{I}_{\mathrm{G}}$ increased with an increase in the growth time and saturated for long growth times. This indicates that the graphene grown on $\mathrm{SiO}_{2}$ grows owing to a surface diffusion reaction; this is the case because of the low solubility of carbon in $\mathrm{SiO}_{2}$. A high $\mathrm{I}_{2 \mathrm{D}} / \mathrm{I}_{\mathrm{G}}$ ratio was realized only when the $\mathrm{H}_{2}$ concentration was low (Figure. 2c). The free carbon dangling bonds tend to terminate in hydrogen, causing $\mathrm{sp}^{2}$ to $\mathrm{sp}^{3}$ transitions. A higher $\mathrm{H}_{2}$ concentration may result in an increase in the amount of amorphous carbon formed rather than in the number of graphitic $\mathrm{sp}^{2}$ structures formed (Figure. 2c). The growth-temperature dependence curve, shown in (Figure. 2a), also exhibited interesting characteristics. A high $\mathrm{I}_{2 \mathrm{D}} / \mathrm{I}_{\mathrm{G}}$ ratio was obtained only at high temperatures $\left(975^{\circ} \mathrm{C}\right)$. (Figure. 2e), shows the optimal growth temperatures for different growth methods.
In general, the energy required for the catalyst-free growth of graphene is higher than that necessary for growth involving the use of a catalyst. It is also known that the optimal growth temperature for nanocarbon materials such as carbon nanotubes [14-16] and graphene [7,8] under plasma CVD is lower than that for thermal CVD growth; this is owing to the high density of reactive species created by the plasma. The experimental results shown in (Figure. 2a) are consistent with this model. A relatively higher temperature $\left(975^{\circ} \mathrm{C}\right)$ is required to realize the catalyst-free growth of graphene compared to that for graphene growth by plasma CVD using a catalyst (600-900) (Figure. 2a). It is likely that the catalyst-free growth of graphene by thermal CVD would be feasible at much higher temperatures $\left(\sim 1200^{\circ} \mathrm{C}\right)$ as well. However, the $\mathrm{SiO}_{2}$ substrate would be damaged at such high temperatures. Thus, compared to other methods, plasma CVD is better suited for the catalyst-free growth of graphene on a $\mathrm{SiO}_{2}$ substrate.

\section{Conclusions}

We realized the catalyst-free growth of high-quality graphene by plasma CVD on $\mathrm{SiO}_{2}$ substrate. Raman spectroscopy mapping 
revealed that the D-peak intensity of the synthesized graphene was lower than the detection limit. A high 2D/G intensity ratio $(\sim 10)$ was obtained from the monolayered graphene area. These results indicated that the defect and impurity densities of the graphene synthesized by the catalyst-free method were very low. Through systematic investigations, it was also found that a relatively high temperature and low $\mathrm{H}_{2}$ concentration were critical for realizing the catalyst-free growth of graphene.

\section{Acknowledgements}

This work was supported in part by Grants-in-Aid for Scientific Research (KAKENHI) grant (25706028) from the Japan Society for the Promotion of Science (JSPS), the OzawaYoshikawa Memorial Electronics Research Foundation, and the Iketani Science and Technology Foundation.

\section{References}

1. Novoselov, K. S., Geim, A. K., Morozov, S. V., Jiang, D., Zhang, Y., Dubonos, S. V., et al. (2004). Electric field effect in atomically thin carbon films. Science, 306(5696), 666-669.

2. Zhang, Y., Tan, Y. W., Stormer, H. L., \& Kim, P. (2005). Experimental observation of the quantum hall effect and berry's phase in graphene. Nature, 438(7065), 201-204.

3. Lin, Y. M., Dimitrakopoulos, C., Jenkins, K. A., Farmer, D. B., Chiu, H. Y., Grill, A., et al. (2010). 100-GHz transistors from wafer-scale epitaxial graphene. Science, 327(5966), 662.

4. Kim, K. S., Zhao, Y., Jang, H., Lee, S. Y., Kim, J. M., Kim, K. S., et al (2009). Large-scale pattern growth of graphene films for stretchable transparent electrodes. Nature, 457(7230), 706-710.

5. Dong, X., Shi, Y., Huang, W., Chen, P., \& Li, L. J. (2010). Electrical detection of DNA hybridization with single-base specificity using transistors based on CVD-grown graphene sheets. Adv. Mater, 22(14), 1649-1653.

6. Reina, A., Jia, X., Ho, J., Nezich, D., Son, H., Bulovic, V., et al. (2009). Large area, few-layer graphene films on arbitrary substrates by chemical vapor deposition. NanoLett, 9(1), 30-35.

7. Kato, T., \& Hatakeyama,R. (2012). Direct growth of doping-densitycontrolled hexagonal graphene on $\mathrm{SiO}_{2}$ substrate by rapid-heating plasma CVD. ACS Nano, 6(10), 8508-8515.

8. Kato, T., \& Hatakeyama,R. (2012). Site- and alignment-controlled growth of graphene nanoribbons from nickel nanobars. Nature Nanotechnology, 7(10), 651-656.

9. Zhang, L., Shi, Z., Wang, Y., Yang, R., Shi, D., \& Zhang, G. (2011). Catalystfree growth of nanographenefilms on various substrates. Nano Res. $4(3), 315-321$.

10. Kim, H., Song, I., Park, C., Son, M., Hong, M., Kim, Y., et al. (2013). Copper-vapor-assisted chemical vapor deposition for high-quality and metal-free single-layer graphene on amorphous $\mathrm{SiO}_{2}$ substrate. ACS Nano, 7(8), 6575-6582.

11. Dresselhaus, M. S., Jorio, A., Hofmann, M., Dresselhaus, G., \& Saito, R. (2010). Perspectives on carbon nanotubes and graphene Raman spectroscopy. NanoLett, 10(3), 751-758.

12. Li, X., Cai, W., Colombo, L., \& Ruoff, R. S. (2009). Evolution of graphene growth on $\mathrm{Ni}$ and $\mathrm{Cu}$ by carbon isotope labeling. NanoLett., 9(12), 4268-4272.

13. Li, X., Cai, W., An, J., Kim, S., Nah, J., Yang, D., et al. (2009). Large-area synthesis of high-quality and uniform graphene films on copper foils. Science, 324(5932), 1312-1314.

14. Kato, T., \& Hatakeyama, R. (2008). Kinetics of reactive ion etching upon single-walled carbon nanotubes. Appl Phys Lett, 92(3), 031502.

15. Kato, T., \& Hatakeyama, R. (2010). Direct growth of short singlewalled carbon nanotubes with narrow-chirality distribution by timeprogrammed plasma chemical vapor deposition. ACS Nano, 4(12), 7395-7400.

16. Ghorannevis, Z., Kato,T., Kaneko,T., \& Hatakeyama,R. (2010). Narrowchirality distributed single-walled carbon nanotubes growth from nonmagnetic catalyst. J. Am. Chem. Soc, 132(28), 9570-9572. 